\title{
METHODS OF MONITORING OF WASTE WATER TREATMENT EFFICIENCY
}

\author{
Lijana Augulyte \\ Per-Anders Bergqvist', ${ }^{2}$ \\ Audrone Zaliauskiene ${ }^{2}$ \\ ${ }^{\prime}$ Umeå University, Sweden \\ ${ }^{2}$ Exposmeter $A B$, Sweden
}

\begin{abstract}
One major result of implementing the Water Frame Directive (WFD) for the water industry is the likelihood of more stringent requirements for the efficiency of removal of the pollutants included in the list of WFD priority substances. During the last decade, an ongoing debate on the technical aspects of water treatment and on the fate and effects of its constituents after discharge are taking place. Due to the recent development of analytical techniques, the knowledge about the chemistry and toxicology of the waste water has increased considerable. Characterization of the oil treatment efficiency by total petroleum hydrocarbon concentration (TPH) is not sufficient any more. Waste water before and after treatment should be characterized by toxicity and amounts of the individual compounds and mixtures which trigger the toxicity.
\end{abstract}

In the situations where more efficient oil removal from waste water is required to meet the requirements, there are a number of treatment options available, including some tertiary ("polishing") treatment systems. New generation tertiary wastewater treatment system for removal or reducing of the oil compounds, including dissolved polycyclic aromatic hydrocarbons, bearing along most toxic potential, was developed under a Eureka funded project. The new waste water treatment system involves novel monitoring device to verify that the dissolved and bioavailable fraction of the contaminants are degraded, and that the final effluent is cleaner also from a toxicity point of view. Previous studies showed that more toxic compounds might be produced during the treatment process due to the transformation of the original chemicals.

The monitoring device consists of a membrane-based diffusive, time integrative (2-30 days) sampler. The newly developed fast analytical method for the analysis of membrane extracts enable to give us information on the dissolved concentrations for more than 80 oil related compounds in the effluents as well as the toxicity results, by using standard bio-assay tests. Moreover the monitoring system is capable to accurately sample most of the WFD priority substances in waste water treatment effluent waters. It was found that some of the compounds were effectively removed in waste water treatment plant (WWTP), but other compounds remained in the dissolved phase at the same concentrations.

Furthermore, volatilization of low molecular weight PAHs during the treatment process was studied by means of the new monitoring system, resulting in the identification of significant release of WFD pollutants to the air. The changes in time-integrative toxicity during the 
treatment process were evaluated by standard ecotoxicity analysis using the same membrane extracts. The three bio-assays used were Daphnia magna, Microtox and Algal but also other organism- or cell test systems will be tested further.

Our developed monitoring device is the state-of-art method for screening for environmental toxicity by integrating biologically and chemically based techniques for early warning and ecosystem health assessment purposes. It can provide invaluable information in highly polluted environments where bioindicator organisms would not survive or behave normally.

\section{KEYWORDS}

Waste water; Monitoring; Cleaning efficiency; Passive sampling; Toxicity

\section{INTRODUCTION}

Wastewater contains many constituents and impurities arising from diffuse and point sources. Over 6,000 organic compounds have been detected in raw water sources during the last decades. Most efforts have been focused on reducing the traditional parameters such as BOD, nitrogen and phosphorus in the effluents because European rivers and lakes show eutrophication symptoms up to $40 \%$. [2] In the new society situation, waste water treatment plants should also take care about compounds and mixtures which can enhance the toxicity of the effluent water as well as of the sludge. The European Commission has proposed a list of 32 priority and 11 hazardous substances (COM/2001/17) with the aim of progressively reducing emissions and discharges of these chemicals to the environment. The organic pollutants of interest in urban waste water are polyaromatic hydrocarbons (PAHs), Polychlorinated byphenyls (PCBs), di(2-ethylhexyl) phthalate (DEHP), linear alkyl benzene sulphonates (LAS), nonyl phenol (NP), dioxins and furans etc.

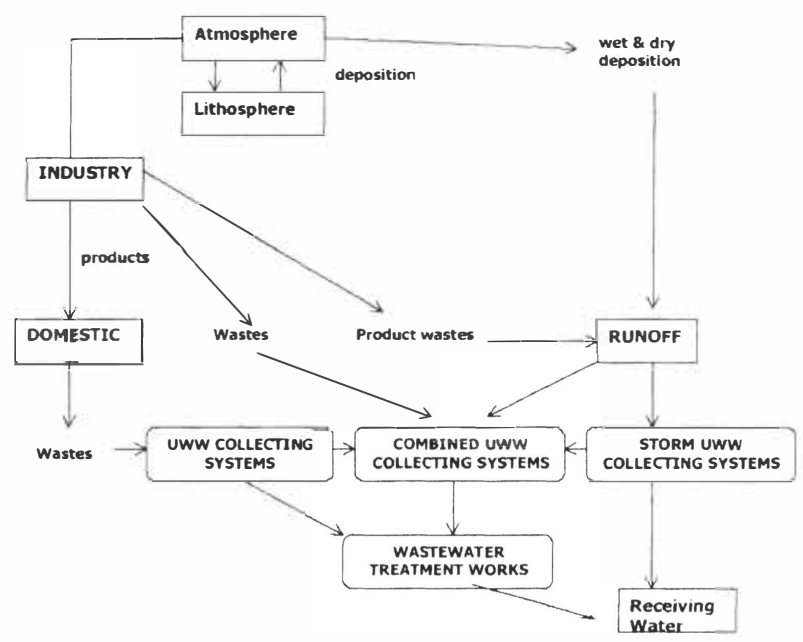

Figure 1 Sources of pollutants in waste water [1]. 
While some of these are highly persistent, others are easily biodegradable or biotransformed in waste water treatment system. Potentially toxic and hydrophobic organic contaminants partly transfer to the sewage sludge during waste water treatment. Increasing body of scientific evidence suggests that substantial amount of them also emitted with effluent waters and has potential harmful impact on the environment $[3,4,5]$. It might result in the future requirement for tertiary treatment processes (e.g. ozonation or some other technology) to eliminate these substances from the effluents. However, the control of those substances in WWTP is still scarce, maybe due to high cost and special analytical requirements needed for quantifying these compounds in sludge and effluent.

Waste water treatment plants has to be equipped with tools for representative samples of the incoming waste water and of treated effluents in order to describe the treatment efficiency for also these specific compounds before the acceptable discharge to receiving waters can be made. The most common technique for carrying out monitoring of waste water treatment efficiency is spot sampling followed by a laboratory-based extraction and determination of compounds of interest. Flow-proportional or time-based 24-hour samples shall be collected at a well-defined point in the outlet and if necessary in the inlet of the treatment plant in order to monitor compliance with the requirements for discharged waste water laid down in Urban waste water treatment directive [6].

During the last years it is undergoing discussion to characterize WWTP effluents not by the total amounts of pollutants analysed originating from grab or composite samples, but from the fraction which might be ecologically relevant or primarily bioavailable. We describe new system named Water Treatment Monitoring Device (WTMD) suitable for purposes of chemical and toxicity testing of waste water cleaning efficiency. This system has been designed for monitoring of all lipophilic organic pollutants included in WFD priority list to meet future requirement for the monitoring of bioavailable concentrations of organic pollutants. The method has not shown any saturation problems also when exposed to remarkable contaminated samples. For hydrophilic compounds, another similar sampler which is optimised for hydrophilic compounds (POCIS), can be used in the same manner.

\section{SYSTEM DESIGN AND METHODOLOGY}

WTMD system can be used both for monitoring of waste water cleaning efficiency in-line and in the receiving water body.

In-line WTMD system consists of the preconcentration media sealed inside the diffusive membrane (semipermable membrane device SPMD [7]) enclosed into a stainless steel protective cage. The restriction membrane allows only truly dissolved or vapor phase organic contaminants i.e., primarily bioavailable residues to diffuse into membrane and accumulate in the media. Hardware configuration allows using system in elevated pressure and temperature conditions. WTMD was tested in a tertiary batch treatment system. The device remained in the water for 7 days, and sampling was performed in water before and after treatment. One device was deployed in the ventilation tube from the aerated treatment reactor. Enclosed membrane was extracted with dialysis after retrieval of WTMD. Half of the extract was analysed for 44 PAHs. Another half of the extract was tested using 3 bioassay tests: Algal (Desmodesmus subspicatus), Bacteria (Vibrio fischeri) "Microtox ${ }^{\circledR}$ ", Crustacean (Daphnia Magna). 
For the application in receiving water the monitoring hardware is designed as perforated cage for good water exchange and moderation of high flow situations reaching the membrane surface. It is also crucial to protect the sampler from ultraviolet light.

\section{RESULTS AND DISCUSSION}

Our present study on efficiency of removal of oil compounds from the waste water is the part of several year investigations on application of diffusive, integrative devices for monitoring of emerging toxic compounds in the effluents and receiving waters. Results of our earlier studies have indicated that some of the potential toxic compounds remained in the dissolved or bioavailable phase at the same concentrations in the effluents after waste water treatment process. More toxic compounds might be produced during the treatment process due to the transformation of the original chemicals. A study in Umeå (Sweden) WWTP have indicated that not all the persistent organic pollutants entering the wastewater treatment is efficiently removed during the cleaning process, even if BOD is efficiently decreased [8]. Dissolved concentrations of some persistent organic pollutants (POPs) even increased. For example dissolved concentrations of "heavy" PAHs increased 3-4 times during the treatment process (Figure 2).

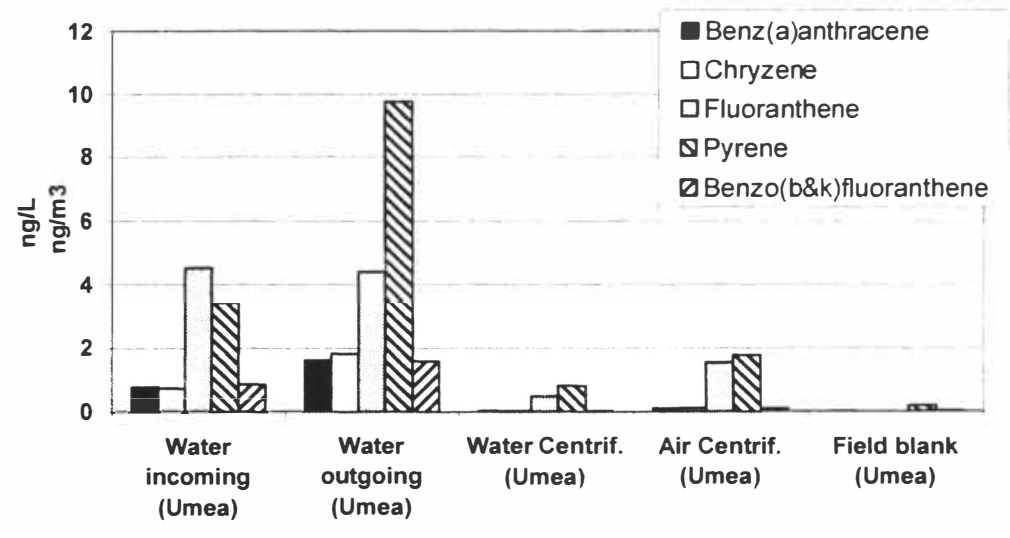

Figure 2. "Heavy" PAHs in Umeå WWTP (Sweden) [8].

Air contaminations by PAHs were identified during the sludge centrifugation process.

\subsection{Monitoring of treatment efficiency}

Tertiary waste water treatment system was taken as the model to validate application of membrane for monitoring of dissolved fraction of $44 \mathrm{PAHs}$ in the effluents. Membranes were deployed before and after tertiary treatment for one week period. After retrieval the SPMD membranes were analysed for sum of 44 PAHs. Three toxicity tests were applied to the extract from the same membrane. Results are presented in Figure 3 and 4. Data in figure 4 is normalized on incoming water concentration for respective compounds. 
Results have verified that the dissolved and bioavailable fraction of sum of 44 PAHs was degraded during the treatment process. However, toxicity to algae in the final effluent has increased. Results suggest that more toxic compounds might be produced during the treatment process due to the transformation of the original chemicals causing toxicity.
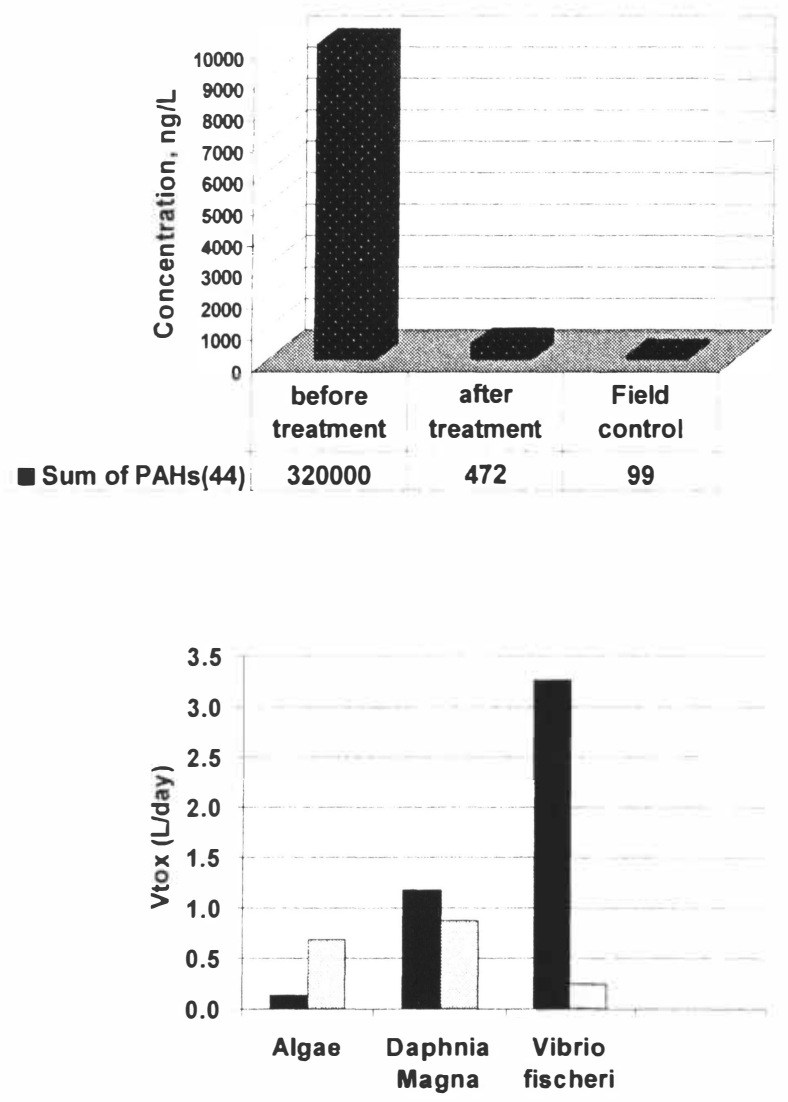

Figure 3. Efficiency of PAHs (44) removal and reduction the eco-toxicity measured with WTMD system. Dark bar represents sample before treatment and light bar represents sample after tertiary treatment step. 


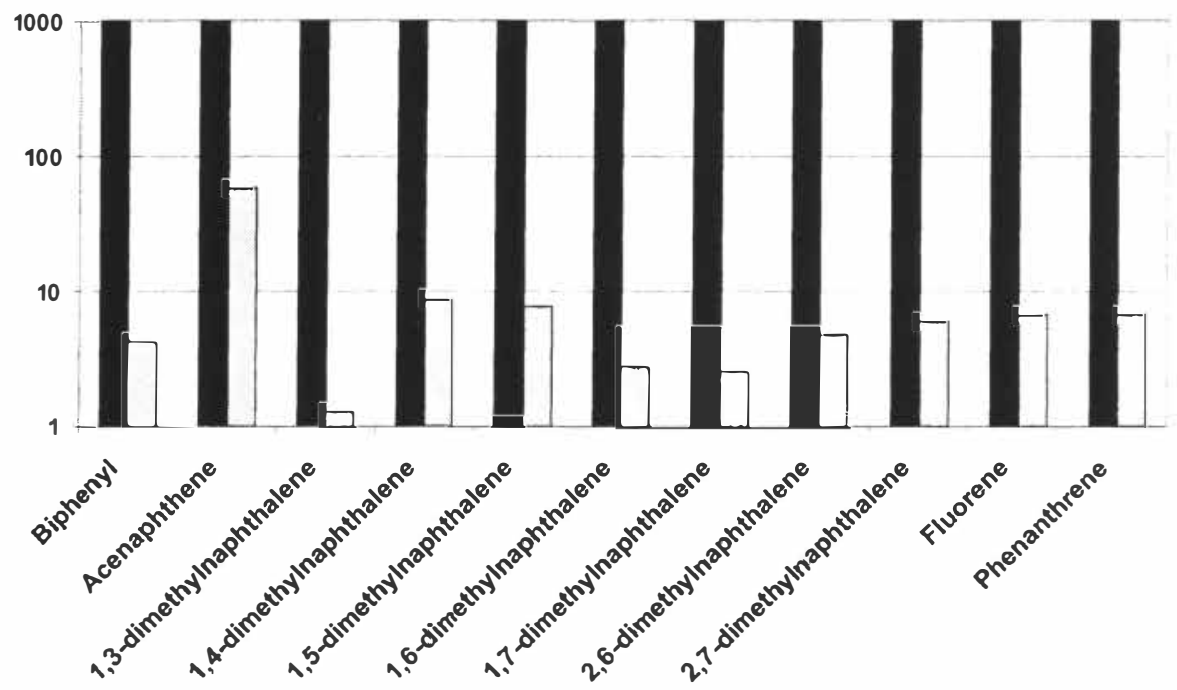

Figure 4. Removal of medium molecular weight PAHs during the treatment process. Data is normalized on incoming water concentration for respective compounds and presented on a logarithmic scale. Dark bar represents sample before treatment and light bar represents sample after tertiary treatment step.

Integrative sampling system can also be applied for further monitoring of exposure, fate and concentrations of toxic compounds discharged from waste water treatment plant to the receiving waters [9].

\section{CONCLUSION}

Integrative monitoring system WTMD based on SPMD technology is a suitable tool for passive sampling of the dissolved part of WFD priority lipophilic pollutants in the high load pollution environment as municipal wastewater. The monitoring system is able to sequester for example PAHs (44) from WWT effluents and to perform suitable sampling for whole effluent toxicity (WET) testing. A time-integrative sampling program can give useful information for risk assessment in connection to chronic exposure effects. The reduction of certain chemical concentrations is far better than the reduction of toxicity during the waste water treatment. Substantial amount of contaminants are released via the ventilation system during the treatment process.

\section{REFERENCES}

[1t] Pollutants in urban waste water and sewage sludge, European Communities, 2001, ISBN92-894-1735-8.

[2] Report from the commission to the council, the European parliament, the European economic and social committee and the committee of the regions Implementation of 
Council Directive 91/271/EEC of 21 May 1991 concerning urban waste water treatment, as amended by Commission Directive 98/15/EC of 27 February 1998, Brussels, 23.4.2004 COM(2004) 248.

[3] Pham, T. T., Proulx, S., 1997. PCBs and PAHs in the Montreal Urban Community (Quebec, Canada) wastewater treatment plant and in the effluent plume in the St Lawrence River. Water Research 31(8): 1887-96.

[4] Rowland, S., et al., 2001. Aromatic hydrocarbon "humps" in the marine environment: Unrecognized toxins? Environmental Science \& Technology 35(13): 2640-44.

[5] Sabaliunas, D., Lazutka, J. R., Sabaliuniene, I., 2000. Acute toxicity and genotoxicity of aquatic hydrophobic pollutants sampled with semipermeable membrane devices. Environmental Pollution 109(2), 251-65.

[6] Urban waste water treatment Directive 91/271/EEC

[7] Huckins, J., Petty, J., Prest, H., Clark, R., Alvarez, D., Orazio, C., Lebo, J., Cranor, W., Johnson, B., 2002. A guide for the use of semipermeable membrane devices (SPMDs) as samplers of waterborne hydrophobic organic contaminants. American Petroleum Institute publication 4690, American Petroleum Institute, Washington, DC.

[8] Bergqvist P-A., Augulyte L., Zaliauskiene, A., 2002. Persistent organic pollutants (POP) in wastewater treatment plants sampled by semipermeable membrane devices (SPMD). Abstract SETAC Europe 12th Annual Meeting 2002, Vienna Austria.

[9] Zaliauskiene A.. Jegorova I., Bergqvist, P-A., 2002. Dissolved organochlorine and PAH pollution profiles in Lithuanian and Swedish waters. Abstract SETAC Europe 12th Annual Meeting 2002, Vienna, Austria. 